\title{
LINEAR PULSATION PERIODS OF THE POST-AGB STARS
}

\author{
M.TAKEUTI, R. TAKANO and S.TAMURA \\ Astronomical Institute, Tôhoku University, Sendai, Japan
}

There may exist stars during and just after the asymptotic giant branch (AGB) stage. V441 (89) is a candidate star. Fernie and Sasselov (1989) have discussed the evolutionary changes of the pulsation periods based on those of the effective temperature, to compare those with observational data. They used Worell's theorical results (Worell 1986) and have found that the observational changes of the period and the colors are too small compared with the theorical values. To examine the effect of opacities on the pulsations periods, we calculate the periods based on new opacities (OPAL, Iglesias and Rogers 1991), and compare them with the data reported previously.

We ignore convection through calculations. The OPAL opacities are used with the chemical composition of population II $(\mathrm{Y}, \mathrm{Z})=(0.299,0.001)$.

To compare the results with those of Fernie and Sasselov (1989), we choose the effective temperature of $5888 \mathrm{~K}\left(\log T_{\text {eff }}=3.77\right)$ for the models. the relation between the period, $\Pi$, and the radius, $R$, is calculated. The gradient of the $\log R-\log \Pi$ relation is determined for the period of 65 days: $\log \Pi=1.813$ (Fernie 1991) The gradient of the $\log R-\log \Pi$ relation is less steep for pulsationally unstable models. For the convenience of comparison, $d T_{\text {eff }} / d t=10 \mathrm{~K} \mathrm{yr}^{-1}$ is chosen. Results are tabulated in table I. The rate labelled by Worrell are found in Fernie and Sasselov (1989). The changes of opacities affect the pulsation period only a little bit.

The combination of new results on stellar evolution and our pulsation properties will yield a new criterion to check the stellar evolution theory by using observational data on variable stars.

TABLE I

Theorical Period Change Rates

\begin{tabular}{rrr}
\hline$M / M_{\odot}$ & $d \Pi / d t$ (Worrell) & $d \Pi / d t$ (Present) \\
\hline 0.644 & -0.24 & -0.24 \\
0.600 & -0.11 & -0.13 \\
0.565 & -0.07 & -0.20 \\
\hline
\end{tabular}

\section{References}

Fernie J.D. 1991, PASP, 105, 1087

Fernie J.D. and Sasselov D.D. 1989, PASP, 101, 513

Iglesias C.A. and Rogers F.J. 1991, Ap.J., 371, L73

Worell J.K. 1986 M.N.R.A.S., 223, 787 